\title{
Tibiakopf: Repositions- und Osteosynthesetechniken
}

Florian Wichlas, Thomas Freude

\section{Einleitung}

Tibiakopffrakturen sind eine heterogene Frakturgruppe. Circa 65\% dieser schweren Gelenkverletzungen der unteren Extremität entstehen als Folge aus sog. High-EnergyTraumen durch Sport- oder Verkehrsunfälle [1]. Eine saisonale Häufung von Tibiakopffrakturen ist durch Wintersport, insbesondere durch das alpine Skifahren, zu beobachten [2,3]. Bei den übrigen $25 \%$ handelt es sich um Low-Energy-Traumen im fortgeschrittenen Alter. Hierbei kann man eine Zunahme durch Trendsportarten, insbesondere E-Bike-Aktivität, beobachten [4-6]. Die Frakturmorphologie beinhaltet einfache Frakturen des lateralen Plateaus (AO 41 B1) bis zu komplexen bikondylären Frakturen (AO 41 C3) mit begleitendem Kompartmentsyndrom oder gleichzeitiger Gefäßverletzung, die eine komplexe Versorgung benötigen. Die Versorgungsstrategien richten sich nach der Schwere der Fraktur und den physiologischen Voraussetzungen des jeweiligen Patienten. Die Therapie reicht von konservativer Therapie über minimalinvasive, perkutane Verfahren [7, 8] bis hin zur gestaffelten „Versorgungsstrategie“ mit primärer externer Fixierung und eventueller Dermatofasziotomie gefolgt von "second“- und „third-look“-Operationen und definitiver Osteosynthese über 2 operative Zugänge mit mehreren Platten $[9,10]$.

Neben der kritischen klinischen Kontrolle der Weichteile ist die CT-Diagnostik, mit oder ohne Gefäßdarstellung, unabdingbar in der Versorgung von Tibiakopffrakturen (CT).

Ziel bleibt die anatomische, möglichst stufenfreie Wiederherstellung der Kontinuität der Gelenkoberfläche in Verbindung mit einer möglichst hohen Primärstabilität, um eine frühfunktionelle Nachbehandlung und die bestmöglichen Voraussetzungen eines Knorpelerhalts zu ermöglichen [11]. Nur unter dieser Prämisse kann die posttraumatische Arthrose des Kniegelenkes vermieden oder zumindest zu verzögert werden.

\section{Vorbereitung}

Zwei wesentliche Behandlungspfade in der Versorgung von Tibiakopffrakturen hängen von der Frage ab, ob es sich um ein Niedrig- oder Hochrasanztrauma handelt. Während das Erste nach initialer Ruhigstellung eine frühe operative Versorgung zulässt, zieht das Zweite eine angepasste Versorgung nach sich ( $\bullet$ Abb. $\mathbf{1}$ ).

Die Endstrecke beider Pfade ist die definitive Osteosynthese der Tibiakopffraktur (offene Reposition und interne Fixierung: ORIF).

Um eine anatomische Reposition erreichen zu können, sind ein schonendes Weichteilmanagement, eine exakte Frakturanalyse und die Wahl des operativen Zugangs mit Planung der zur Verfügung stehenden Implantate unerlässlich [12-15]. Ein wesentlicher Faktor für eine erfolgreiche Behandlung einer Tibiakopffraktur ist auch der gewählte Zeitpunkt der Versorgung. Der Zeitpunkt dieser u.U. aufwendigen Operation darf keinesfalls zu früh erfolgen. Komplexe Frakturen führen oft zu erheblichen Weichteilschäden.

Cave

Aufgrund des hohen operativen Komplikationsrisikos sollte die definitive Versorgung nur bei absolut unauffälligen Weichteilen erfolgen!

\section{Lagerung}

Eine sorgfältige Patientenlagerung erleichtert die Reposition, ermöglicht eine gute Erreichbarkeit des Frakturherdes und verkürzt dadurch die Operationszeit. Wir empfehlen 2 Lagerungen: Rücken- oder Bauchlagerung. Verschiedene Keilkissen, Lagerungsrollen und Kippungen des Operationstisches sollten unbedingt zur Unterstützung verwendet werden, auch während der Operation bei kombinierten Zugängen ( $\triangleright$ Tab. 1 und 2). Wenn keine Kontraindikationen bestehen, verwenden wir eine Blutsperre.

\section{Zugang}

Der anterolaterale Zugang war lange Zeit der Hauptzugang zum Tibiakopf. Obwohl er immer noch als „AlphaZugang“ zum Tibiakopf bezeichnet werden darf, gibt es heute fast keinen Zugang zum Tibiakopf, der nicht beschrieben ist [9, 16-19].

In der Versorgung von Tibiakopffrakturen, spielt die Wahl der operativen Zugänge eine Schlüsselrolle, mit der sich auch die rezente Literatur beschäftigt. Neue Klassifikationen unterstützen den Operateur in der Entscheidungsfin- 


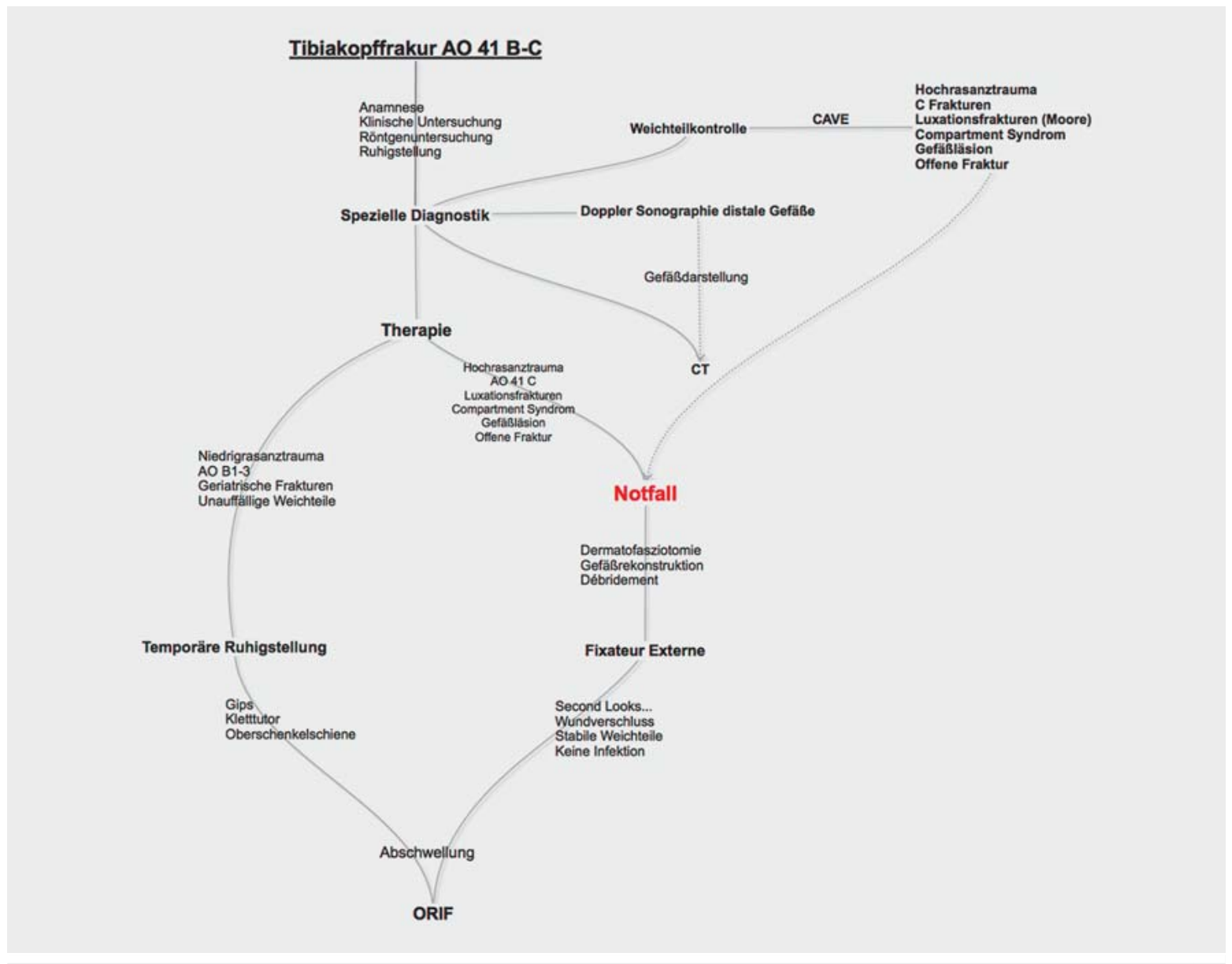

- Abb. 1 Behandlungspfad.

dung für den Zugang und in der optimalen Osteosynthesetechnik in Bezug auf die Frakturmorphologie von Tibiakopffrakturen; es handelt sich hierbei um das 3- bis 4Säulen-Modell oder die 10-Segment-Klassifikation [14, 20,21].

Die Wahl des Zugangs erfolgt durch Analyse des CT. Folgende Faktoren sind zu berücksichtigen:

- Der Frakturherd muss direkt erreichbar sein.

- Der Zugang sollte die Kontrolle von knöchernen Landmarken ermöglichen.

- Die Osteosynthese sollte der Dislokationstendenz der Fraktur(komponente) entgegenwirken.

Schatzker hat über die Jahre 1968-1975 ausführlich den Zusammenhang zwischen Frakturmorphologie, gewähltem Osteosyntheseverfahren und konsekutiver Versagensquote in seiner „Toronto experience“ dargestellt. Daraus hatte er abgeleitet, dass eine Beteiligung sowohl des lateralen wie auch des medialen Tibiaplateaus die Versorgung mittels zweier Platten bedingt; dieses Vorgehen hat heute noch Bestand, auch wenn winkelstabile Implantate eine höhere Primärstabilität ermöglichen [22].
- Tab. 1 Lagerung des Patienten nach gewählten Zugangsweg.

\begin{tabular}{|l|l|}
\hline Lagerung & Zugang \\
\hline Rückenlage & " anterolateral \\
& " posterolateral (transfibulär) \\
& " medial \\
& " posteromedial \\
\hline Bauchlage & " alle posterioren Zugänge \\
\hline & " anterolateral (in floating position) \\
\hline
\end{tabular}

\section{Reposition}

Zwischen dem operativen Zugang und der abgeschlossenen Osteosynthese liegen folgende Schritte:

- Darstellung der Fraktur (Landmarken) mit „Schaffen“ des Plattenlagers

- „Fraktursäuberung“ - Débridement der Frakturenden

- Reposition

- temporäre Fixierung

- definitive Fixierung 
- Tab. 2 Hilfsmittel zur exakten Lagerungskontrolle und Unterstützung beim Repositionsmanöver.

\begin{tabular}{|c|c|}
\hline Zugang & Lagerung, Lagerungshilfen und Maßnahmen \\
\hline $\begin{array}{l}\text { antero- } \\
\text { lateral }\end{array}$ & $\begin{array}{l}\text { Rückenlage: Das Bein sollte innenrotiert liegen. } \\
\text { - Keilkissen unter dem gleichseitigen Gesäß } \\
\text { - Rolle unter dem Knie } \\
\text { - Tisch kippen bei Bedarf }\end{array}$ \\
\hline $\begin{array}{l}\text { postero- } \\
\text { lateral }\end{array}$ & $\begin{array}{l}\text { Rückenlage: Das Bein sollte (noch mehr) innenrotiert liegen. } \\
\text { - Keilkissen unter dem gleichseitigen Gesäß } \\
\text { - Rolle unter dem Knie } \\
\text { - Tisch kippen bei Bedarf }\end{array}$ \\
\hline $\begin{array}{l}\text { postero- } \\
\text { medial }\end{array}$ & $\begin{array}{l}\text { Rückenlage: Das Bein sollte nach außen rotiert liegen. } \\
\text { - Außenrotation und Beugen im Kniegelenk („4er-Position“) } \\
\text { - Rolle unter dem Knie } \\
\text { - Rolle unter dem Sprunggelenk (dadurch hängt der } \\
\text { M. gastrocnemius durch) } \\
\text { - Senken des gegenseitigen Beines (zum Bohren) } \\
\text { - bei Bedarf Tisch kippen } \\
\text { - bei Bedarf Keilkissen unter dem gegenseitigen Gesäß }\end{array}$ \\
\hline medial & $\begin{array}{l}\text { Rückenlage: Das Bein sollte nach außen rotiert liegen. } \\
\text { - Rolle unter dem Knie } \\
\text { - bei Bedarf wie posteromedial }\end{array}$ \\
\hline posterior & $\begin{array}{l}\text { Bauchlage } \\
\text { " „mobile Rolle“ unter dem Sprunggelenk oder dem Knie } \\
\text { " Tisch kippen bei Bedarf }\end{array}$ \\
\hline $\begin{array}{l}\text { anterolate- } \\
\text { ral und } \\
\text { postero- } \\
\text { medial }\end{array}$ & $\begin{array}{l}\text { Rückenlage: Wechseln zwischen Innen- und Außenrotation } \\
\text { des Beines } \\
\text { " „mobile Rolle“ } \\
\text { - Tisch kippen während der Operation } \\
\text { " Keilkissen (Gesäß) einschieben während der Operation }\end{array}$ \\
\hline $\begin{array}{l}\text { anterolate- } \\
\text { ral und } \\
\text { posterior }\end{array}$ & $\begin{array}{l}\text { Bauchlage: Floating Position } \\
\text { - Tisch kippen } \\
\text { - zur gleichen Seite den posterioren Zugang } \\
\text { - zur Gegenseite den anterolateralen Zugang } \\
\text { - Knie beugen für anterolateralen Zugang }\end{array}$ \\
\hline
\end{tabular}

Wir unterscheiden die direkte und indirekte Reposition. Während der direkten Reposition werden Frakturanteile genau dargestellt und manipuliert. Die indirekte Reposition lässt die Fraktur (weichteil)gedeckt und die Manipulation erfolgt fern des Frakturherds. Die Reposition des Tibiakopfes erfolgt hauptsächlich per direkter Reposition. Es kommen jedoch beide Techniken zum Einsatz ( $\triangleright$ Tab. 3). Prinzipiell gilt es, während der OP-Schritte aus einer komplexen Fraktur eine einfache zu machen und dann die definitive Fixierung vorzunehmen.

\section{A-Frakturen (extraartikulär)}

Die Reposition der extraartikulären Frakturen, und demnach nicht Tibiakopffrakturen, gleicht jener im Schaftbereich und wird hier nicht behandelt.

\section{B-Frakturen (partiell intraartikulär, ein Gelenk- anteil steht mit dem Schaft in Verbindung)}

Die monokondylären Frakturen werden, abhängig vom betroffenen Kondyl, von anterolateral oder (postero-)medial versorgt.
- Tab. 3 Technische Repositionshilfen und Instrumente.

\begin{tabular}{|c|c|}
\hline Repositionshilfen & Einsatzmöglichkeit \\
\hline Lagerung & indirekte Reposition \\
\hline $\begin{array}{l}\text { Spitz-Spitz-Repositions- } \\
\text { zange (groß/klein) }\end{array}$ & $\begin{array}{l}\text { - direkte Reposition } \\
\text { - temporäre Fixierung }\end{array}$ \\
\hline $\begin{array}{l}\text { Kondylen-Repositionszange } \\
\text { (Kugel-Spitz-Spitz) }\end{array}$ & $\begin{array}{l}\text { - direkte Reposition } \\
\text { - Kompression des Tibia- } \\
\text { plateaus }\end{array}$ \\
\hline Kirschner-Drähte (1-2 mm) & $\begin{array}{l}\text { - direkte Reposition } \\
\text { - temporäre Fixierung } \\
\text { - „Joystickdrähte“ } \\
\text { - Inside-out-Technik }\end{array}$ \\
\hline $\begin{array}{l}\text { Raspatorium, Elevatorium, } \\
\text { Zahnarzthaken, Stößel } \\
\text { (gerade, gebogen, breit, } \\
\text { schmal), Lambotte-Meißel } \\
\text { (breit, schmal) }\end{array}$ & $\begin{array}{l}\text { - direkte Reposition } \\
\text { " „Heben“ der Gelenk- } \\
\text { fläche } \\
\text { - Osteotomie }\end{array}$ \\
\hline Arthrodesenspreizer & $\begin{array}{l}\text { - direkte Reposition } \\
\text { - Öffnen der Fraktur (open } \\
\text { the book) }\end{array}$ \\
\hline OP-Assistent & $\begin{array}{l}\text { - indirekte Reposition } \\
\text { - Traktion } \\
\text { - Varus-/Valgusstress } \\
\text { - Flexion/Extension }\end{array}$ \\
\hline Distraktor & $\begin{array}{l}\text { - indirekte Reposition } \\
\text { - Traktion } \\
\text { - Aufklappen des Gelenks }\end{array}$ \\
\hline
\end{tabular}

Der anterolaterale Zugang führt zum lateralen Plateau. Die submeniskale Arthrotomie erlaubt die visuelle Kontrolle der Gelenkfläche. Sollte sich die Fraktur- oder Impressionszone weit nach posterior erstrecken, kann zusätzlich eine subkapitale Fibulaosteotomie durchgeführt werden (posterolateraler Zugang), um dieses Areal zu sehen.

Reine „Splitfrakturen“ (B1) stützen wir mit einer anatomisch geformten winkelstabilen 3,5-mm-Platte ab. Schrauben von lateral nach medial können bei guter Knochenqualität ebenfalls verwendet werden. Nachdem der Frakturspalt, mittels Arthrodesenspreizer oder breiten Raspatoriums, aufgeklappt wurde (open the book), erfolgt die Fraktursäuberung. Das laterale frakturierte Fragment wird dann angespickt (Joystickdrähte, $2 \mathrm{~mm}$ ) und unter intraartikulärer Kontrolle der Fraktur reponiert. Die Joystickdrähte können dann nach medial vorgetrieben werden, um so die Fraktur temporär zu fixieren. Um die folgende Plattenosteosynthese nicht zu behindern, können die Drähte auch medial perkutan „rausgeschossen“ werden. Eine große Repositionszange (aufgesetzt im Tuberositasbereich) oder eine übergreifende Kondylenrepositionszange (medial über eine Stichinzision aufgesetzt) bringen das Plateau auf Kompression. Die beste Kompression kann mit der Kondylenrepositionszange über die definitive Platte lateral und eine Unterlegscheibe 

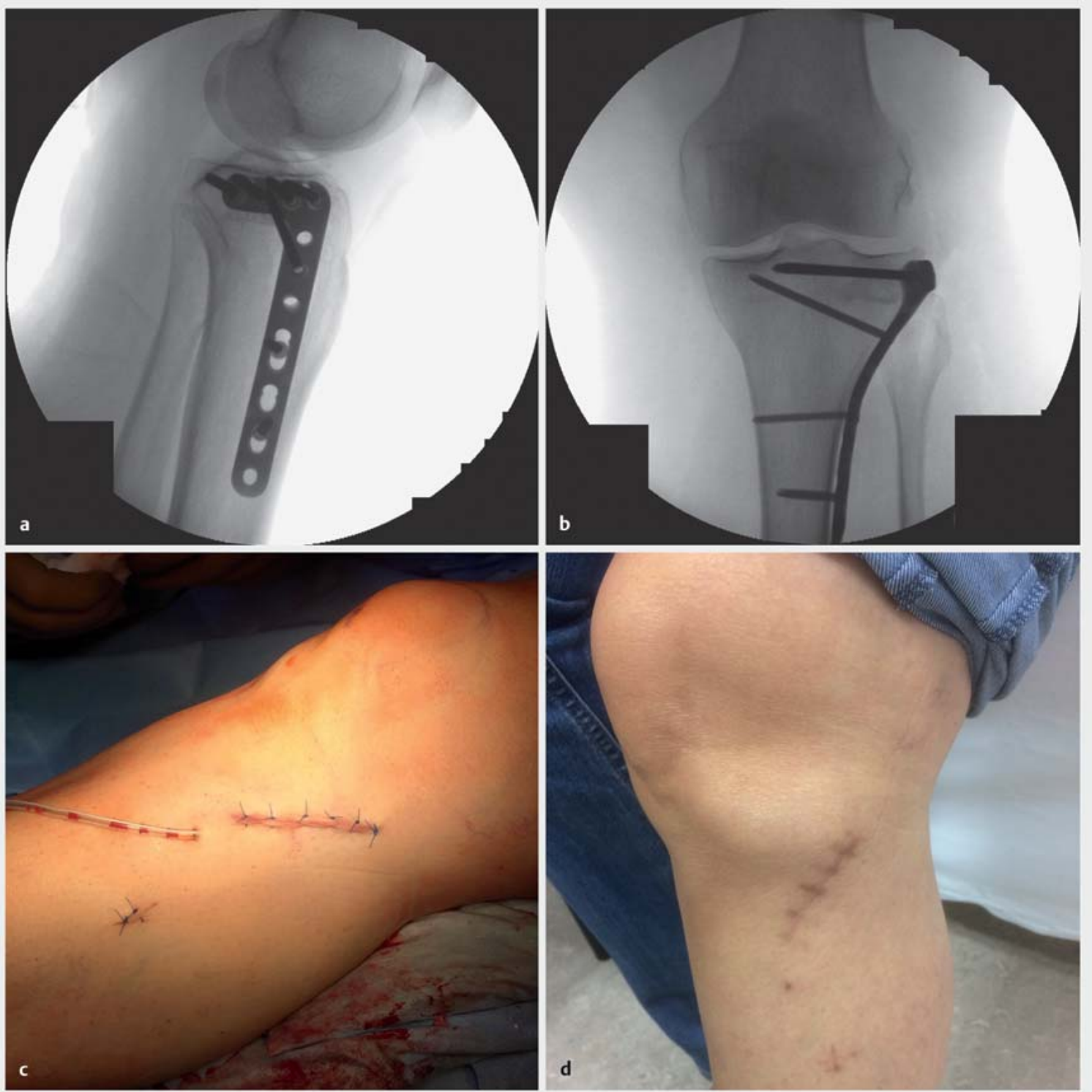

- Abb. 2 Tibiakopffraktur, AO 41 B2 (Impression) laterales Plateau. Offene Reposition und interne Fixierung mit lateraler proximaler Tibia-LCP $\left(\right.$ Synthes $\left.^{\circledR}\right)$ und Spongiosaplastik über einen kleinen anterolateralen Zugang.

(oder eine 3-Loch-Drittelrohrplatte) medial erzeugt werden, ohne das Einbrechen der Zange zu riskieren. Die Platte sollte mit einer plattenabhängigen Zugschraube herangezogen und anschließend winkelstabil verblockt werden. Wir beschränken die Größe des anterolateralen Zugangs auf die Fraktur und den Gelenkspalt (etwa $5 \mathrm{~cm}$ ) und verriegeln die Platte distal über Stichinzisionen ( Abb. 2).

Die Schraubenosteosynthese (6,5-mm-Spongiosaschrauben mit Unterlegscheiben und/oder 3,5-/4,5-mm-Kortikalisschrauben) erfolgt in gleicher Weise, kann aber auch, bei undislozierten Frakturen, perkutan durchgeführt werden.
Impressionsfrakturen (B2), die zentral liegen, können über ein kortikales Fenster „aufgestößelt“ oder, wenn sie peripher liegen, über eine Osteotomie mit dem Meißel angehoben werden. Die visuelle Kontrolle der Reposition erfolgt über die submeniskale Arthrotomie. Beim Aufstößeln ist es wichtig, das Fenster so zu platzieren, dass die imprimierte Gelenkfläche mit dem meist gebogenen Stößel derart erreicht werden kann, dass der Kraftvektor senkrecht zur Gelenkfläche steht. Unser häufigster Fehler war es, das Fenster zu weit proximal osteotomiert zu haben und mit dem Stößel nicht „um die Ecke“ gekommen zu sein.

Die Reposition mittels Kyphoplastieballon stellt eine elegante Alternative dar. 


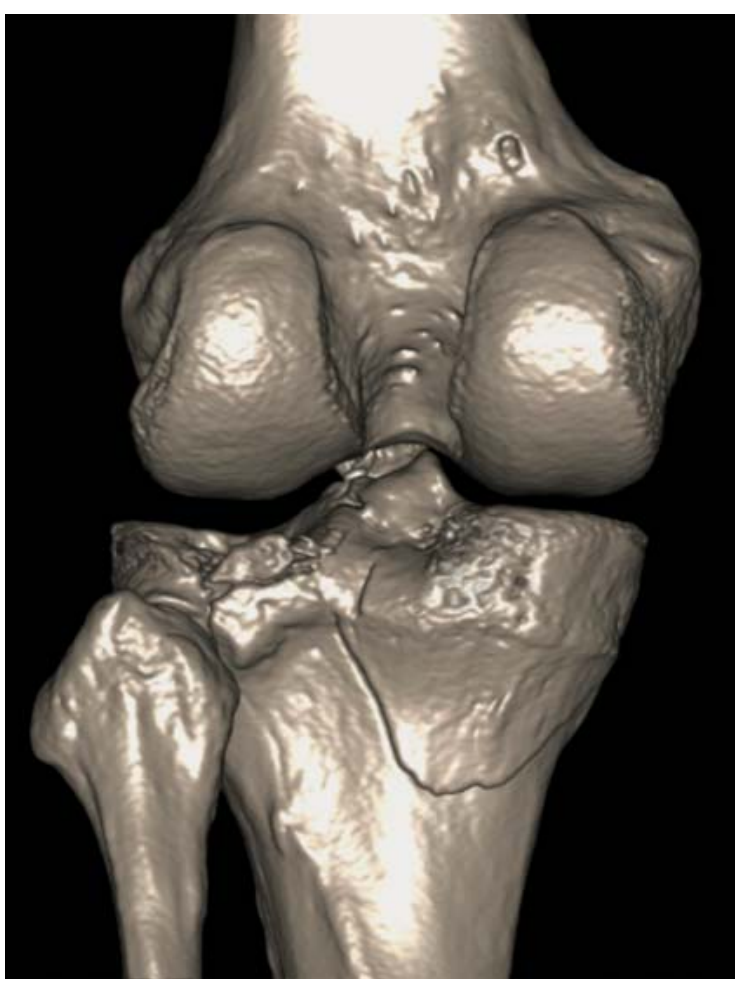

- Abb. 3 Tibiakopffraktur mit posteromedialem Keil und dorsolateraler Impression, CT-3-D-Rekonstruktion.

Bei der klassischen Methode, bei der durch ein geeignetes Instrument das Imprimat anzuheben ist, werden punktuell hohe Kräfte auf das Stratum regenerativum eingeleitet, was zu einer zusätzlichen Schädigung der Gelenkfläche führt.
Die Ballonosteoplastie bietet ein Alternativverfahren, das die Krafteinleitung bei der Reposition punktuell vermindert und gleichzeitig eine anatomische Wiederherstellung des Tibiakopfes ermöglicht [23].

Die OP-Technik unterscheidet sich in der Lagerung und im 1. Teil der Versorgungsstrategie nicht vom Vorgehen bei einem klassischen „anterolateralen“ Zugangsweg. Der Patient wird in Rückenlage auf den für die Durchleuchtung geeigneten OP-Tisch gelagert. Das zu operierende Bein wird in ca. $15^{\circ}$ Flexion im Kniegelenk gebracht, wobei sich hierbei die Verwendung geeigneter steriler Rollen bewährt hat, da diese intraoperativ auch entfernt werden können. In dem gewählten Beispiel handelt es sich um einen Patienten, der eine Tibiakopffraktur mit einer posteromedialen Splitkomponente und einer posterolateralen Impression erlitten hat ( $\bullet$ Abb. $\mathbf{3}$ )

In einem 1. Schritt wird über einen dorsalen Zugang die Reposition des posteromedialen Fragments vorgenommen ( $\triangleright$ Abb. 4).

Im nächsten Schritt werden unter exakter Darstellung im Bildwandler mindestens 2 2,0-mm-K-Drähte von medial ca. $1 \mathrm{~cm}$ unterhalb der Impressionszone platziert ( Abb. 5).

Dieser Schritt ist wichtig, da der Ballon bei höheren Drücken die Tendenz hat, den Weg des geringsten Widerstandes zu wählen. Das heißt, der Ballon weicht nach kaudal in die metaphysären Anteile der proximalen Tibia aus; die K-Drähte wirken diesem Ausweichen entgegen. Im

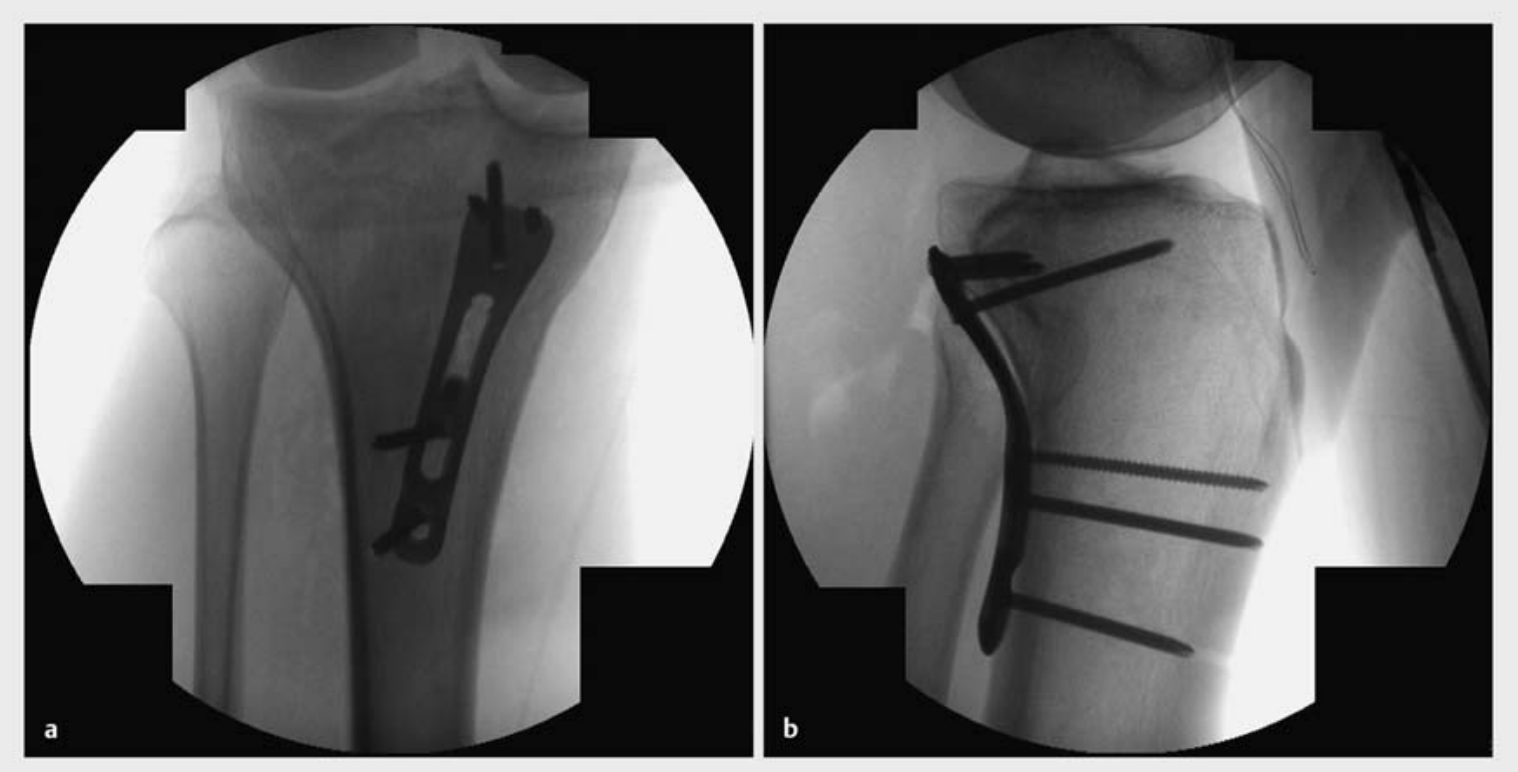

- Abb. 4 Reposition und Fixierung des dorsomedialen Fragments über dorsalen Zugang mit 3,5-mm-Tibia-LCP. 
nächsten Schritt wird der Osteoplastieballon platziert ( $\triangleright$ Abb. 6).

Über die Führungskanüle wird der Ballon eingebracht und schrittweise mit kontrastmittelhaltiger Flüssigkeit befüllt. Die Drücke, die notwendig sind, um eine suffiziente Redressierung des Imprimats zu gewährleisten, sind mit bis zu 350 PSI sehr hoch.

Die Reposition geschieht unter Bildwandlerkontrolle oder über direkte Einsichtnahme ins Gelenk über eine laterale Arthrotomie unter Anheben des Meniscus lateralis ( A Abb. 7).

Es ist darauf zu achten, dass es zu einem gleichmäßigen Anheben des Imprimats kommt und der Ballon nicht ausweicht und damit ein Verkippen des Imprimats bewirkt. Ebenso muss darauf geachtet werden, dass die Splitkomponente nicht durch den Ballon aufgedrückt wird. Um dies zu vermeiden, hat sich die sog. „King-Kong“-Zange bewährt die als Kompressionsinstrument ventral über beide Tibiakondylen aufgesetzt wird und somit ein „Bulging“ des lateralen Tibiakopfes verhindert.

Der geschaffene Hohlraum wird über die Führungskanüle mit Spongiosa oder Knochenersatzmittel befüllt. Bei allen Frakturen mit einer Impression der Gelenkfläche und einem entsprechenden metaphysären Substanzdefekt nach erfolgreicher Anhebung des Imprimats auf Gelenkniveau sollte eine Unterfütterung der Gelenkfläche und Auffüllung des Defektes erfolgen, um einem sekundären Repositionsverlust vorzubeugen. Als Füllmaterial stehen dem Operateur Eigenspongiosa, Fremdspongiosa oder künstliche Kalziumphosphatverbindungen (bspw. Chronos $^{\mathrm{TM}}$, Vitoss ${ }^{\mathrm{TM}}$ ) oder Hydroxylapatitverbindungen (bspw. Endobon $^{\mathrm{TM}}$ ) zur Verfügung.

Die weitere Stabilisierung erfolgt mittels lateraler proximaler Tibia-LC-Platten oder/und mit 6,5-mm-Kurzgewinde-Spongiosazugschrauben ( $\bullet$ Abb. 8).

Die bisher veröffentlichten Arbeiten sind als Erfahrungsberichte oder als Beschreibung der OP-Technik zu verstehen [24-27].

\section{Zu beachten:}

1. Ballonosteoplastie ist geeignet bei mehrfragmentären Impressionszonen.

2. Auf eine exakte Platzierung des Ballons ist zu achten.

3. Der Ballon muss mit K-Drähten unterstützt werden.

4. Die Stabilisierung erfolgt mit Platten oder Schrauben.

Wenn die Impression nahe am Rand des Gelenks liegt, schlagen wir den Meißel unterhalb der Impressionszone ein und heben das Fragment vorsichtig. Der häufigste Fehler dieses Verfahrens ist es, zu nahe am Gelenk zu osteotomieren, wodurch sich das dann sehr kleine Frag-

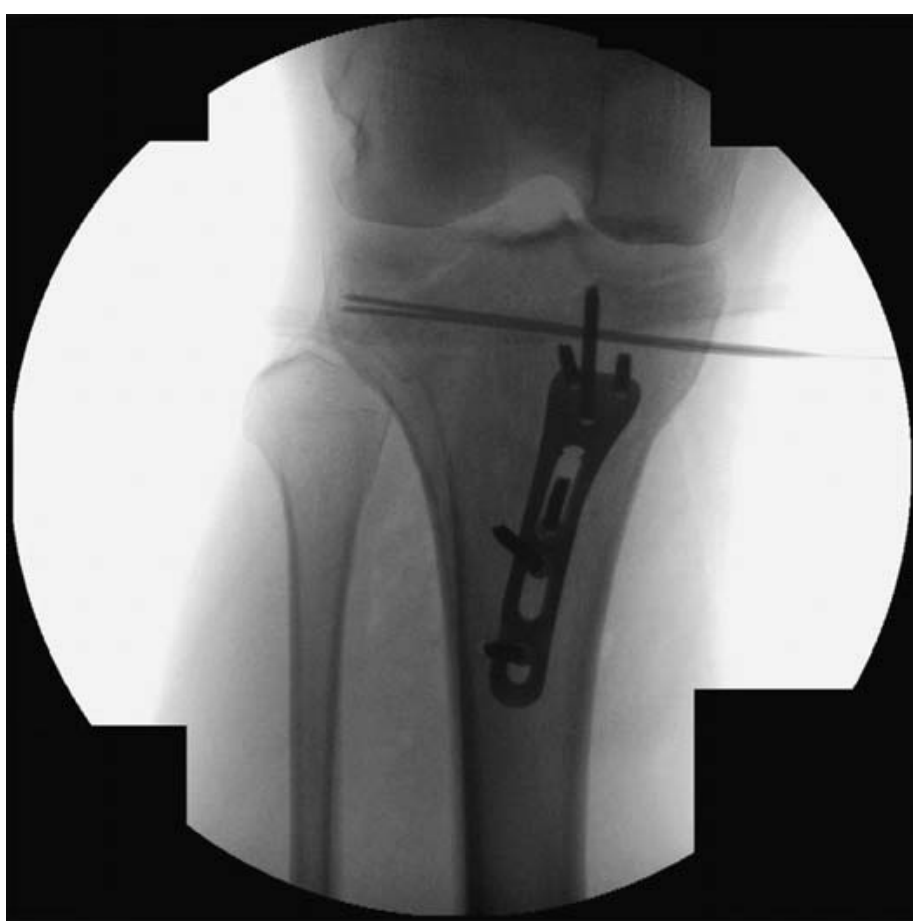

- Abb. 5 Bildwandlerkontrollierte Platzierung von 2 K-Drähten ca. $1 \mathrm{~cm}$ unterhalb der Impressionszone als Widerlager für den Ballon.

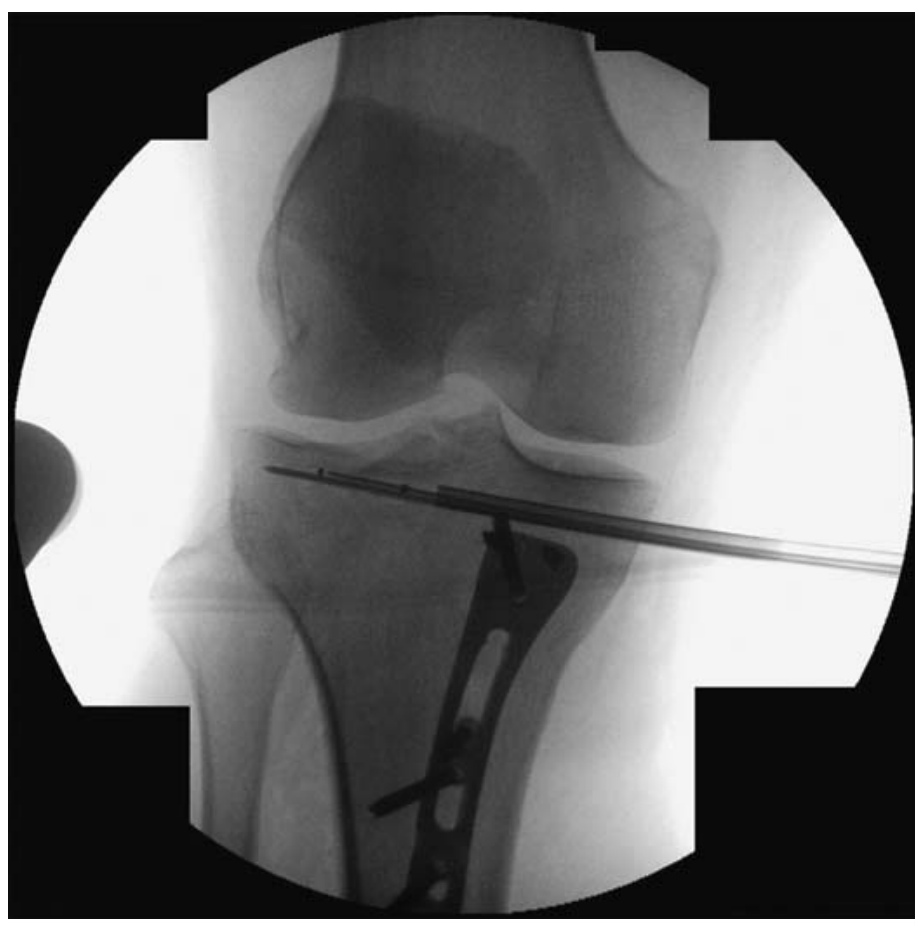

Abb. 6 Platzierung des Ballons. 


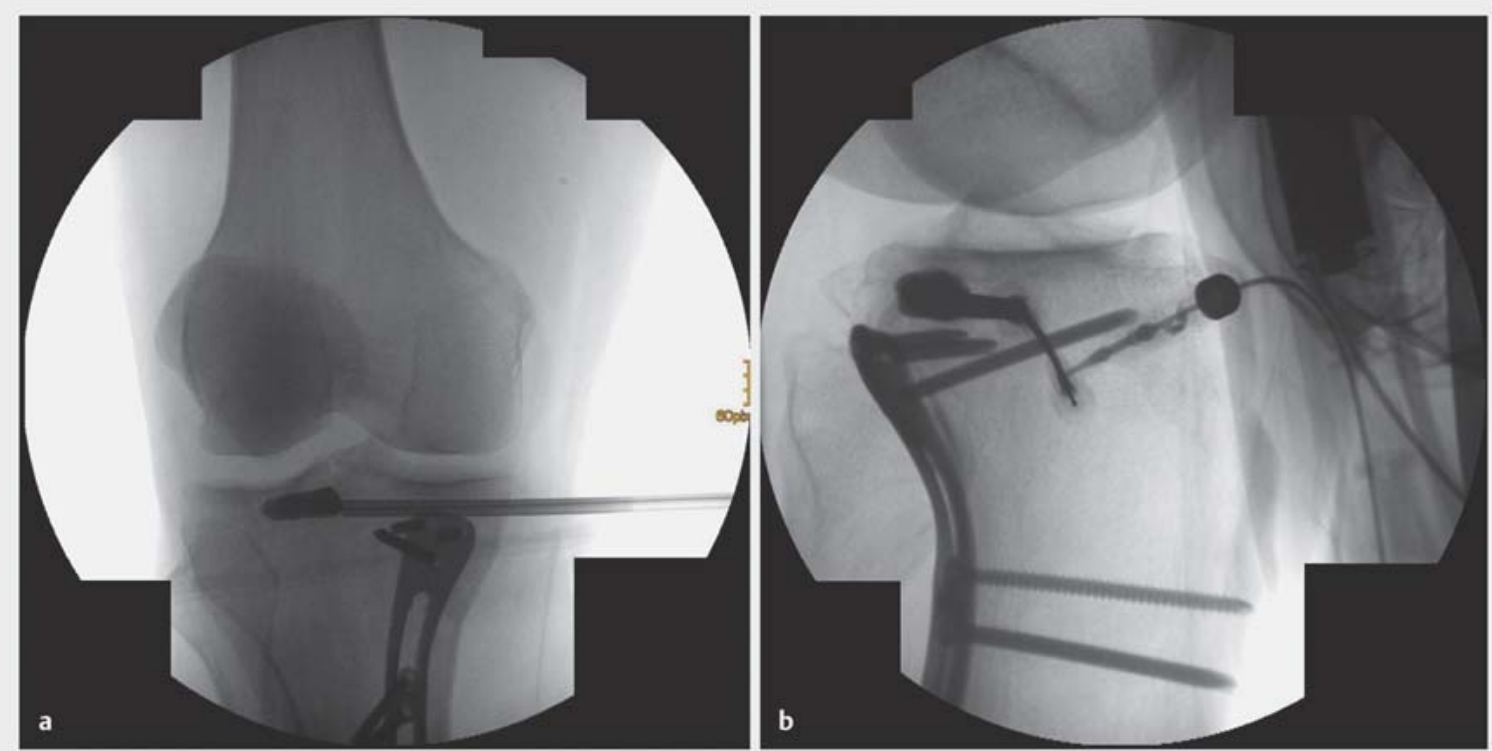

Abb. 7 a Anheben des Imprimats, a.-p. Strahlengang. b Seitliche Darstellung der Platzierung des Ballons und Anheben des Imprimats.
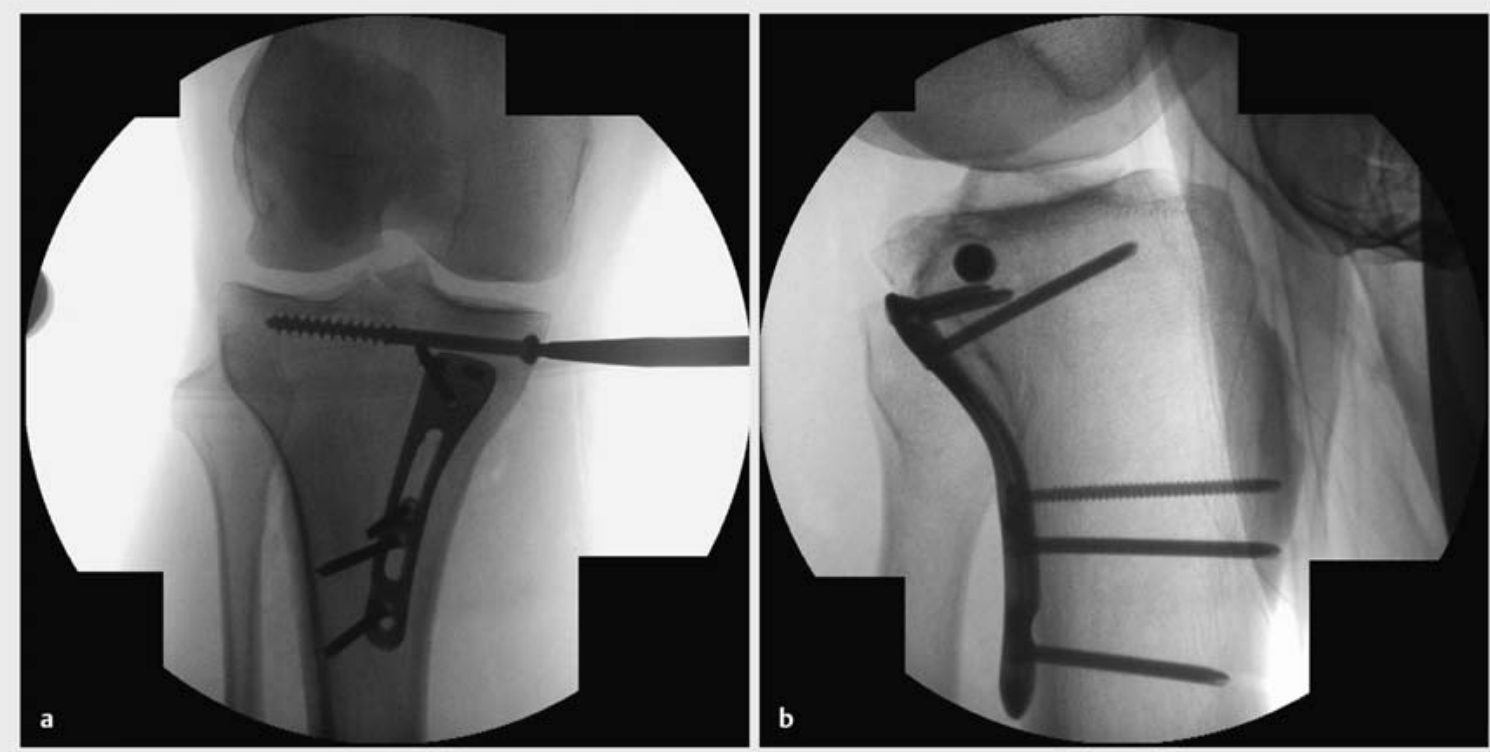

- Abb. 8 Abstützen des angehobenen Imprimats mit Großfragmentspongiosaschraube.

ment löst und nur schwer zu refixieren ist. Der entstandene Osteotomiedefekt kann aufgefüllt werden ( $>$ Abb. 2).

Das angehobene Fragment sollte mit K-Drähten temporär fixiert und mit einer Platte abgestützt werden.
Splitimpressionsfrakturen (B3) werden über eine Kombination o.g. Methoden reponiert. Die „Splitkomponente“ kann, mittels Arthrodesenspreizer, von anterior aufgespreizt (open the book) und die Impressionszone aufgerichtet werden. Die Reposition erfolgt danach analog zu den Splitfrakturen. Manche Autoren empfehlen das 

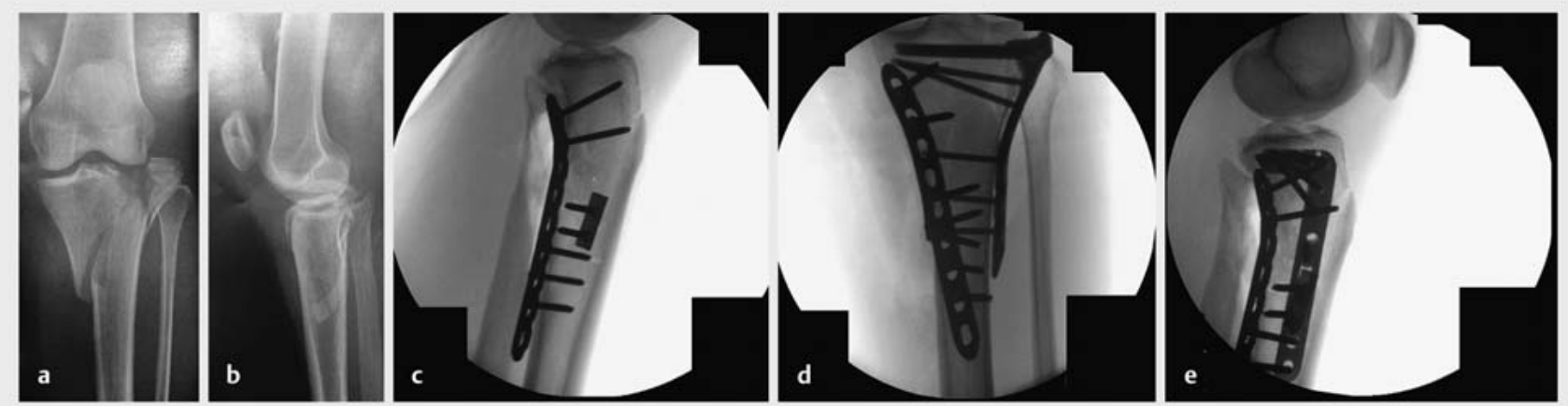

- Abb. 9 Versorgung instabiler Tibiakopffrakturen. Hochgradig instabile Tibiakopfluxationsfraktur (AO 41 C2). Röntgenbild der Fraktur, anteroposterior und seitlich $(\mathbf{a}, \mathbf{b})$. Zum Schutz der Weichteile erfolgt die sofortige Versorgung mit dem Fixateur externe und die kritische Kontrolle der Weichteile (Gefahr eines Kompartmentsyndroms). Bildverstärkerbild intraoperativ nach Anlage des Fixateur externe, anteroposterior und seitlich. Die definitive Versorgung mit offener Reposition und interner Fixierung mit 3-facher Plattenosteosynthese erfolgt, nachdem die Weichteile abgeschwollen sind. Die Fraktur wird über einen posteromedialen und anterolateralen Zugang mit 3 winkelstabilen Platten versorgt: 1 . Anatomische Reposition und Fixierung einer Landmarke mit einer kleinen 2-Loch-Drittelrohr-LCP (Synthes ${ }^{\circledR}$ ): Vereinfachen der Fraktur. 2. Posteromediale Reposition und Abstützung mit 3,5-mm-LCP (Synthes ${ }^{\circledR}$ ). Aus der C2-Fraktur ist nun eine B3-Fraktur geworden (c). 3. Anterolateraler Zugang mit ORIF mit lateraler proximaler 3,5-mm-Tibia-LCP $\left(\right.$ Synthes $\left.{ }^{\circledR}\right)$. Intraoperative Abschlusskontrolle, anteroposterior (d) und seitlich (e).

Aufstößeln der Depressionszone über den Frakturspalt ohne Aufspreizen, um das laterale Frakturfragment nicht zu devitalisieren. Die visuelle und „digitale“ Kontrolle des rekonstruierten Plateaus mittels Elevatorium über die Arthrotomie ist essenziell und macht den Bildverstärkereinsatz erst nach Anlegen der Platte notwendig.

Die Reposition des medialen Plateaus erfolgt in gleicher Weise über einen medialen oder posteromedialen Zugang, abhängig vom Frakturherd. Im Gegensatz zu den „Splitfrakturen“ des lateralen Plateaus, die in der sagittalen Ebene liegen, liegt die Frakturebene des medialen Plateaus meistens in der frontalen Ebene und läuft posterodistal metaphysär aus. Die Reposition erfolgt unter Längszug bei gestreckten Knie unter Zuhilfenahme einer anteroposterioren spitzen Repositionszange. Die Platte sollte dorsal, als Antigleitplatte, angebracht werden. Alternativ dazu, kann „über die Platte“ reponiert werden, indem diese regelrecht positioniert und über eine plattenabhängige Zugschraube angezogen wird. Ein posteriorer Zugang kann für diese Frakturen ebenfalls benützt werden. Hier wird ein Großteil der Reposition bereits durch die Lagerung erreicht und die Besetzung der Schrauben vereinfacht.

\section{C-Frakturen (komplett artikulär, es besteht keine Verbindung zwischen Schaft und Gelenk)}

Diese Frakturen benötigen 2 Zugänge. Wir reponieren und fixieren einen Kondylus zuerst und danach den zweiten mit oben erwähnten Techniken. Manchmal reponie- ren wir gleichzeitig über beide Zugänge. Der einfachere Kondylus sollte zuerst reponiert werden und so gut wie immer handelt es sich dabei um den medialen, da hier klare Landmarken ohne Trümmerzone zu identifizieren sind ( $\bullet$ Abb. 9). Das mediale Plateau hat meistens einen frontalen Frakturverlauf, der, wie oben beschrieben, über einen posteromedialen Zugang reponiert werden kann. Die posteriore Abstützplatte sichert das Ergebnis. Das laterale Plateau wird dann wie eine B-Fraktur, über einen passenden lateralen Zugang, behandelt.

Komplexe Frakturtypen mit Trümmerzonen können eine anatomische Reposition unmöglich machen und das posttraumatische Arthroserisiko erhöhen. Die $>$ Abb. 10 zeigt ein zertrümmertes laterales Plateau einer C3-Fraktur.

Komplexe Frakturtypen mit Trümmerzonen können eine anatomische Reposition unmöglich machen und das posttraumatische Arthroserisiko wesentlich erhöhen. Die - Abb. 10 zeigt ein zertrümmertes laterales Plateau einer C3-Fraktur. Die Reposition solcher Frakturen ist besonders anspruchsvoll und selbst wenn sie perfekt gelingt, kann auch sie das Auftreten einer posttraumatischen Arthrose, aufgrund des irreversiblen Knorpelschadens, nicht verhindern.

\section{Fazit}

Während die Versorgung einfacher Tibiakopffrakturen selten Probleme verursacht, stellen komplexe Luxationsfrakturen eine Herausforderung dar. Die Behandlung ist aufwendig und erfordert viel Erfahrung, da häufig nicht nur der Knochen betroffen ist, sondern auch die Weich- 


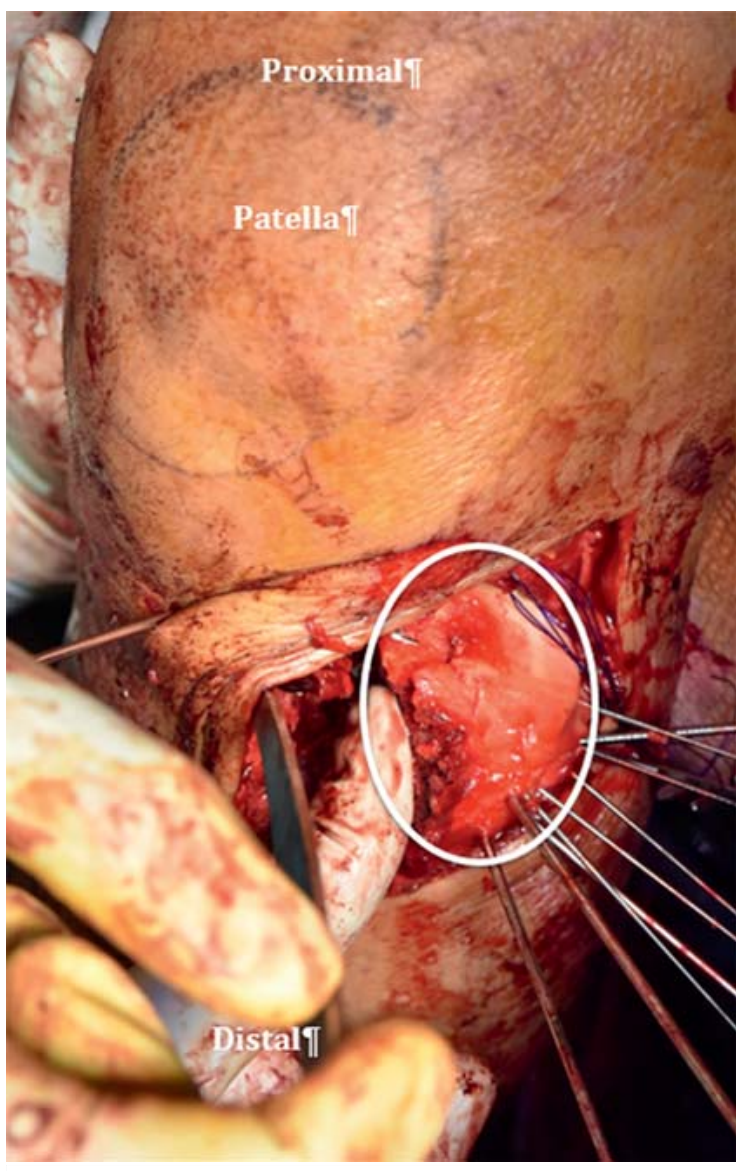

- Abb. 10 Mehrfragmentäres laterales Tibiaplateau. Intraoperativer Situs des lateralen Plateaus (weißer Kreis) über einen anterolateralen Zugang zeigt das Ausmaß des Gelenkschadens. Die multiplen Kirschner-Drähte sind Ausdruck der schwierigen Reposition des lateralen Plateaus. Die Trümmerzone und der Knorpelschaden führen unausweichlich zu einer frühen posttraumatischen $\mathrm{Ar}$ throse.

teile. Trümmerzonen, die eine anatomische Reposition unmöglich machen, anatomische Areale der Gelenkfläche, die schwer erreichbar sind und das hohe Komplikationsrisiko führen dazu, dass diese Frakturen von erfahrenen Operateuren versorgt werden sollten.

Die Kombination aus einer exzellenten präoperativen Bildgebung, einer sich aus dieser ergebenden Frakturanalyse und OP-Planung und das Wissen um aktuelle OP-Verfahren ist der Schlüssel zu einer guten Versorgung von Tibiakopffrakturen. Zur Prädiktion des langfristigen Outcomes sind 2 Faktoren entscheidend. Zum einen die Frakturschwere $[1,28]$, insbesondere das Ausmaß der Gelenkbeteiligung [29]; zum Zweiten die exakte intraoperative anatomische Reposition [30,31]. Zusammenfassend müssen jedoch gemäß aktueller Literatur für B3-/C3Frakturen die schlechtesten Ergebnisse für die klinischen Scores erwartet werden $[3,6]$.
Interessenkonflikt

Die Autoren geben an, dass kein Interessenkonflikt besteht.

Autorinnen/Autoren

\section{Florian Wichlas}

Priv.-Doz. Dr., Oberarzt, Universitätsklinik für Orthopädie und Traumatologie, Paracelsus Medizinische Privatuniversität Salzburg

\section{Thomas Freude}

Univ.-Prof. Dr., Vorstand der Universitätsklinik für Orthopädie und Traumatologie der PMU, Universitätsklinik für Orthopädie und Traumatologie, Paracelsus Medizinische Privatuniversität Salzburg

Korrespondenzadresse

\section{Florian Wichlas, MD}

Universitätsklinik für Orthopädie und Traumatologie Paracelsus Medizinische Privatuniversität Salzburg Müllner Hauptstr. 48

5020 Salzburg

Österreich

Tel.: + 43/(0)5/7255-50001

Fax: $+43 /(0) 5 / 7255-50004$

f.wichlas@salk.at

\section{Literatur}

[1] Berkson EM, Virkus WW. High-energy tibial plateau fractures. J Am Acad Orthop Surg 2006; 14: 20-31

[2] Müller D, Sandmann GH, Martetschläger F et al. [Tibial plateau fractures in alpine skiing-return to the slopes or career end?]. Sportverletz Sportschaden 2014; 28: 24-30

[3] Loibl M, Bäumlein M, Massen F et al. Sports activity after surgical treatment of intra-articular tibial plateau fractures in skiers. Am J Sports Med 2013; 41: 1340-1347

[4] Meisinger C, Wildner M, Stieber ] et al. [Epidemiology of limb fractures]. Orthopade 2002; 31: 92-99

[5] Mardian S, Schwabe P, Schaser KD. [Fractures of the tibial shaft]. Z Orthop Unfall 2015; 153: 99-119

[6] Kraus TM, Martetschläger F, Müller D et al. Return to sports activity after tibial plateau fractures: 89 cases with minimum 24month follow-up. Am J Sports Med 2012; 40: 2845-2852

[7] Gill T], Moezzi DM, Oates KM et al. Arthroscopic reduction and internal fixation of tibial plateau fractures in skiing. Clin Orthop Relat Res 2001; (383): 243-249

[8] Stuby F. Arthroskopisch assistierte Osteosynthese bei Tibiakopffrakturen. Trauma Berufskrankheit 2008; 10 (Suppl. 3): 3366-3369

[9] Frosch KH, Balcarek P, Walde T et al. [A modified posterolateral approach for the treatment of tibial plateau fractures]. Oper Orthop Traumatol 2010; 22: 107-119

[10] Acklin YP, Potocnik P, Sommer C. Extended medial approach in posteromedial proximal tibia fracture dislocation. Oper Orthop Traumatol 2014; 27: 183-190 
[11] Stevens DG, Beharry R, McKee MD et al. The long-term functional outcome of operatively treated tibial plateau fractures. J Orthop Trauma 2001; 15: 312-320

[12] Reul M, Hoekstra H. [Combined posteromedial and anterolateral approach in the "floating position" - two column fracture of the tibia head]. Z Orthop Unfall 2017; 155: 661-669

[13] Cho JW, Kim J, Cho WT et al. Approaches and fixation of the posterolateral fracture fragment in tibial plateau fractures: a review with an emphasis on rim plating via modified anterolateral approach. Int Orthop 2017; 41: 1887-1897

[14] Luo CF, Sun H, Zhang B et al. Three-column fixation for complex tibial plateau fractures. J Orthop Trauma 2010; 24: 683692

[15] Cho JW, Samal P, Jeon YS et al. Rim plating of posterolateral fracture fragments (PLFs) through a modified anterolateral approach in tibial plateau fractures. J Orthop Trauma 2016; 30: e362-e368

[16] Lobenhoffer P, Gerich T, Bertram T et al. [Particular posteromedial and posterolateral approaches for the treatment of tibial head fractures]. Unfallchirurg 1997; 100: 957-967

[17] Galla M, Riemer C, Lobenhoffer P. [Direct posterior approach for the treatment of posteromedial tibial head fractures]. Oper Orthop Traumatol 2009; 21: 51-64

[18] Carlson DA. Posterior bicondylar tibial plateau fractures. J Orthop Trauma 2005; 19: 73-78

[19] Sun DH, Zhao Y, Zhang JT et al. Anterolateral tibial plateau osteotomy as a new approach for the treatment of posterolateral tibial plateau fracture: a case report. Medicine (Baltimore) 2018; 97: e9669

[20] Krause M, Preiss A, Müller G et al. Intra-articular tibial plateau fracture characteristics according to the "Ten segment classification". Injury 2016; 47: 2551-2557

[21] Wang Y, Luo C, Zhu Y et al. Updated Three-Column Concept in surgical treatment for tibial plateau fractures - a prospective cohort study of 287 patients. Injury 2016; 47: 1488-1496

[22] Schatzker J, McBroom R, Bruce D. The tibial plateau fracture. The Toronto experience 1968-1975. Clin Orthop Relat Res 1979; (138): 94-104
[23] Ahrens P, Sandmann G, Bauer ] et al. Balloon osteoplasty - a new technique for reduction and stabilisation of impression fractures in the tibial plateau: a cadaver study and first clinical application. Int Orthop 2012; 36: 1937-1940

[24] Mauffrey C, Fader R, Hammerberg EM et al. Incidence and pattern of technical complications in balloon-guided osteoplasty for depressed tibial plateau fractures: a pilot study in 20 consecutive patients. Patient Saf Surg 2013; 7: 8

[25] Pizanis A, Garcia P, Pohlemann T et al. Balloon tibioplasty: a useful tool for reduction of tibial plateau depression fractures. J Orthop Trauma 2012; 26: e88-e93

[26] Hahnhaussen J, Hak DJ, Weckbach $S$ et al. Percutaneous inflation osteoplasty for indirect reduction of depressed tibial plateau fractures. Orthopedics 2012; 35: 768-772

[27] Werner CM, Scheyerer M], Schmitt J et al. [Minimally invasive balloon-assisted reduction and internal fixation of tibial plateau fractures]. Unfallchirurg 2012; 115: 1126-1132

[28] Barei DP, Nork SE, Mills W] et al. Functional outcomes of severe bicondylar tibial plateau fractures treated with dual incisions and medial and lateral plates. J Bone Joint Surg Am 2006; 88: 1713-1721

[29] Markhardt BK, Gross JM, Monu JU. Schatzker classification of tibial plateau fractures: use of CT and MR imaging improves assessment. Radiographics 2009; 29: 585-597

[30] Manidakis N, Dosani A, Dimitriou R et al. Tibial plateau fractures: functional outcome and incidence of osteoarthritis in 125 cases. Int Orthop 2010; 34: 565-570

[31] Tscherne H, Lobenhoffer P. Tibial plateau fractures. Management and expected results. Clin Orthop Relat Res 1993; (292): $87-100$

\section{Bibliografie}

DOI https://doi.org/10.1055/a-0761-2567

Online-publiziert 23.01.2019 | OP-JOURNAL 2019; 35: 118127 @ Georg Thieme Verlag KG Stuttgart · New York ISSN 0178-1715 